Research Paper

\title{
Kartogenin regulates hair growth and hair cycling transition
}

\author{
Yuhong Chen"\#, Lijuan zhou 2\#, Yuxin Ding1, Xiaoshuang Yang'1, Jing Jing1, Xianjie Wu ${ }^{1}$, Jufang Zhang ${ }^{3}{ }^{\boxplus}$, \\ Zhongfa Lu1 ${ }^{\boxplus}$ \\ 1. Department of Dermatology, The Second Affiliated Hospital, School of Medicine, Zhejiang University, Hangzhou 310009, PR China. \\ 2. Department of Dermatology, Huashan Hospital, Fudan University, 12 Middle Wulumuqi Road, Shanghai 200040, PR China. \\ 3. Department of cosmetic \& plastic surgery, Affiliated Hangzhou First People's Hospital, Zhejiang University School of Medicine, Add: 261 Huansha road, \\ Hangzhou 311100, PR China. \\ \#These authors contributed equally to this work.
}

$\square$ Corresponding authors: Jufang Zhang, Department of cosmetic \& plastic surgery, Affiliated Hangzhou First People's Hospital, Zhejiang University School of Medicine, Add: 261 Huansha road, Hangzhou 311100, PR China, E-mail: zhjuf@vip.sina.com. Zhongfa Lu, Department of Dermatology, The Second Affiliated Hospital, Zhejiang University School of Medicine, Add: 88 Jiefang Road, Hangzhou 310009, PR China, E-mail: lzfskin@zju.edu.cn.

(c) The author(s). This is an open access article distributed under the terms of the Creative Commons Attribution License (https://creativecommons.org/licenses/by/4.0/). See http://ivyspring.com/terms for full terms and conditions.

Received: 2021.10.25; Accepted: 2022.02.23; Published: 2022.03.06

\begin{abstract}
Background: Kartogenin is a heterocyclic compound able to promote the proliferation, migration, and differentiation of various cell types and induce cartilage-like tissue regeneration. However, the role of kartogenin in hair follicles (HFs), remains unknown. We therefore investigated the effects of kartogenin on the regulation of hair growth and hair growth cycle transition.

Methods: The effects of kartogenin on the proliferation, cell cycle status, and migration of primary human outer root sheath cells (ORSCs) were evaluated by MTS assay, flow cytometry, Transwell ${ }^{\circledR}$ and scratch assays, respectively. We exposed ORSCs to kartogenin $(1 \mu \mathrm{M})$ and determined changes in mRNA and protein levels of transforming growth factor (TGF)- $\beta 2 / \mathrm{Smad}$ signaling molecules by reverse transcription polymerase chain reaction, western blotting, and immunofluorescence. We also examined the effects of kartogenin $(10 \mu \mathrm{M})$ on HFs in mice by histology following cutaneous injection.

Results: Kartogenin enhanced ORSC proliferation and migration function in a dose-dependent manner, and downregulated the expression of TGF- $32 / S m a d$ signaling molecules in vitro. Injection of kartogenin delayed catagen phase and increased regenerated hair length in mice in vivo.

Conclusions: Kartogenin modulates HF growth and regulates the hair cycle and the TGF- $\beta 2 / \mathrm{Smad}$ signaling pathway, providing a potential new approach for the treatment of hair loss.
\end{abstract}

Key words: Kartogenin, Hair growth, Hair follicle, Outer root sheath cell, Transforming growth factor- $\beta$ signaling

\section{Introduction}

The hair growth cycle includes three phases: a growth phase (anagen phase), regression phase (catagen phase), and quiescence phase (telogen phase) [1]. During anagen phase, epithelial proliferation occurs together with thickening, elongation, and pigmentation of the hair shaft, while catagen phase is characterized by rapid apoptosis, and telogen is a relatively quiescent phase [2]. Hair loss (alopecia) affects the sufferer's appearance and can have serious effects on their mental health [3]. Androgenic alopecia (AGA) is the most common type of hair loss, characterized by a shorter anagen phase and miniaturization of hair follicles (HFs) [1,4]. Current therapies for alopecia are thus mainly based on extending the anagen phase and enlarging the hair bulb [5]. However, existing drug therapies have some limitations [6]. Finasteride is only useful for alopecia caused by abnormal androgens (increased conversion from testosterone to dihydrotestosterone), and may have side effects associated with androgen blockage, while its usen in women is still limited $[7,8]$. Although minoxidil acts as a widely used clinical drug for AGA, the effects are not permanent and require long term topical use of the medication [9]. There is thus an 
urgent need to find more effective and safe methods for treating hair loss.

Kartogenin is a recently synthesized small heterocyclic compound $[10,11]$ shown to promote the proliferation, migration, and differentiation of various cell types [12] such as chondrocyte, mesenchymal stem cells, and to promote cartilage-like tissue regeneration $[13,14]$. Decker et al. demonstrated that kartogenin increased chondrocyte proliferation, migration, and differentiation via activation of the hedgehog and transforming growth factor (TGF)- $\beta$ signaling pathways [15]. However, to the best of our knowledge, the function of kartogenin in regulating hair growth and the HF cycle remains unclear.

Outer root sheath cells (ORSCs) act as a reservoir for various epithelial stem cell populations and play a critical role in the maintenance and development of the HF. ORSCs are regarded as precursor cells of interfollicular epidermal keratinocytes [16] and play a critical role in skin stem cell biology and matrix replenishment $[17,18]$.

The functions of ORSCs are regulated by various signaling molecules, among which the transforming growth factor (TGF)- $\beta 2$ is crucial [19-21]. TGF- $\beta 2$, which is a multifunctional cytokine, propagates signals via TGF- $\beta$ receptor and phosphorylating Smad 2 and Smad3 [22]. Various studies demonstrated that TGF- $\beta 2$ is a catagen phase inducer and inhibition of TGF- $\beta 2$ activity during hair cycle could suppress the transition from anagen phase to catagen phase and prolong anagen phase [19].

In this study, we investigated the function of kartogenin in hair growth and HF cycle regulation. The results increased our understanding of the role of kartogenin in the regulation of hair growth and the HF cycle, and may provide a potential new approach for the treatment of hair loss.

\section{Materials and methods}

All protocols for handling of human tissue were approved by the Zhejiang University School of Medicine Second Affiliated Hospital Institutional Review Board.

\section{Isolation and culture of normal ORSCs from human scalp}

We followed the methods of Lijuan Zhou et al. 2018 [23,24]. Scalp specimens were obtained from healthy human ( $\mathrm{n}=15 ; 10$ females and 5 males; age range, 20-50 years) who were undergoing cosmetic surgery, with informed consent. ORSCs were isolated from the scalp specimens by two-step enzyme digestion. ORSCs were centrifugation at $800 \times g$ for 10 min and then resuspended in defined keratinocyte serum-free medium supplemented with keratinocyte growth factor (Gibco, Invitrogen, USA). ORSCs were cultured at $37^{\circ} \mathrm{C}$ and $5 \% \mathrm{CO}_{2}$. The medium was changed twice a day. Cells were passaged at $70-80 \%$ confluence and were used in passage four for the following experiments.

\section{Cell proliferation assay}

The cells were seeded into 96-well plates at the concentration of $1.5 \times 10^{4} /$ well. After cultured with SFM supplemented with kartogenin at $0,0.5$, or $1 \mu \mathrm{M}$ for $24 \mathrm{~h}, 20 \mathrm{\mu l}$ 3-(4,5-dimethylthiazol-2-yl)-5-(3carboxymethoxyphenyl)-2-(4-sulfophenyl)-2H-tetrazo lium (MTS) (Promega) was added to each well at the last $30 \mathrm{~min}$ of incubation. The wavelength of $490 \mathrm{~nm}$ was used to measure light absorption value of each well by ELX808 microplate reader (BioTek, Winooski, VT, USA).

\section{Cell cycle analysis by flow cytometry}

After kartogenin treatment $(0$ or $1 \mu \mathrm{M})$ for $24 \mathrm{~h}$, cells were re-suspended in PBS and were subsequently fixed in $75 \%$ pre-cooled ethyl alcohol for $8 \mathrm{~h}$ at $4{ }^{\circ} \mathrm{C}$. Cells were then centrifuged for $5 \mathrm{~min}$ at $1000 \mathrm{rpm}$, rinsed trice with PBS, dyed with propidium iodide-RNase using a commercial kit (BD Biosciences, San Jose, CA, USA), according to the manufacturer's protocol. The stained cells were analyzed by BD FACSCaliburTM flow cytometer (BD Biosciences, San Jose, CA) and data was analyzed using CytExpert software. Data were presented by percentage of cells compared to the control group.

\section{Transwell ${ }^{\circledR}$ migration assay}

$200 \mathrm{uL}$ ORSCs cells $\left(1 \times 10^{6} / \mathrm{mL}\right)$ were suspended in basic keratinocyte medium without keratinocyte growth factor and plated into the upper chamber. The lower chamber was added with $500 \mathrm{uL}$ SFM containing 0 or $1 \mu \mathrm{M}$ kartogenin. Following $24 \mathrm{~h}$ of incubation at $37{ }^{\circ} \mathrm{C}$, the invasive cells were fixed with $4 \%$ paraformaldehyde for $20 \mathrm{~min}$ at room temperature and stained with crystal violet for $5 \mathrm{~min}$. Stained cells were visualized and counted using an inverted microscope (Olympus, Tokyo, Japan) in five randomly selected fields of view.

\section{Wound healing assay}

ORSCs $\left(2 \times 10^{5}\right.$ cells/well $)$ were plated into a 6-well plate and cultured until they reached $100 \%$ confluency. A $200-\mu l$ sterile pipette tip was subsequently used to make a vertical scratch. The cells were then washed with PBS three times to remove non-adherent cells and incubated with SFM supplemented with or without $(0$ or $1 \mu \mathrm{M})$ for $24 \mathrm{~h}$. The migration distances were captured after 0,12 , and kartogenin $24 \mathrm{~h}$ using a microscope (Olympus, Tokyo, 
Japan) and assessed using Adobe Photoshop CS3 software (Adobe Inc., San Jose, CA, USA).

\section{Reverse transcription-polymerase chain reaction ( $R T-P C R$ )}

After kartogenin treatment $(0$ or $1 \mu \mathrm{M})$, total RNA was extracted using TRIzol reagent (Ambion, New York, NY, USA). Reverse transcription and RT-PCR was performed as described previously $[23,24]$. The sequences of the specific primers (Sangon Biotech, Shanghai, China) used for RT-PCR are listed in Table 1. The mRNA expression levels of target genes were described relative to that of GAPDH using the $2^{-\Delta \Delta \mathrm{Ct}}$ method.

Table 1. Sequences of primers used for RT-PCR

\begin{tabular}{lll}
\hline Gene & Primer & Sequence \\
\hline GAPDH & Forward & 5'-CTCACCGGATGCACCAATGTT-3' \\
& Reverse & 5'-CGCGTTGCTCACAATGTTCAT-3' \\
TGF- 32 & Forward & 5'-CATCCCGCCCACTTTCTAC-3' \\
& Reverse & 5'-AATCCGTTGTTCAGGCACTC-3' \\
Smad2 & Forward & 5'-CCGACACACCGAGATCCTAAC-3' \\
& Reverse & 5'-GAGGTGGCGTTCTGGAATATAA-3' \\
Smad3 & Forward & 5'-CCATCTCCTACTACGAGCTGAA-3" \\
& Reverse & 5'-CACTGCTGCATTCCTGTTGAC-3' \\
\hline
\end{tabular}

\section{Western blot analysis}

The protocol for western blot has been described previously $[23,24]$. Primary antibodies were used as follows: rabbit polyclonal anti-TGF- $\beta 2$ (1:250; Santa Cruz Biotechnology, Santa Cruz, CA, USA), and rabbit polyclonal anti-phosphorylated Smad2 (p-Smad2)/phosphorylated Smad3 (p-Smad3) (1:1000; Cell Signaling Technology, Danvers, MA, USA). GAPDH detected with rabbit monoclonal anti-GAPDH antibody (1:1000; Cell Signaling Technology, MA, USA) served as the loading control.

\section{Immunofluorescence}

Cells were fixed with $4 \%$ paraformaldehyde for 15 min and washed three times with cold PBS, then blocked with $10 \%$ fetal bovine serum for $1 \mathrm{~h}$ at room temperature. $0.1 \%$ Triton X-100 was used for blocking for $10 \mathrm{~min}$. ORSCs were then incubated with TGF- $\beta 2$ (Abcam, Cambridge, MA, USA; 1:200), p-Smad2 and p-Smad3 (Cell Signaling Technology; 1:200) antibodies overnight $(16 \mathrm{~h})$ at $4{ }^{\circ} \mathrm{C}$. After incubation with fluorescence-conjugated secondary antibody (Jackson Laboratories; 1:200) for $2 \mathrm{~h}$ in the dark, nuclei were stained with DAPI (1:5000; Roche, China) for 5 min. Protein localization and expression in ORSCs were compared by fluorescence microscopy (EU5888; Leica, Wetzlar, Germany) and four randomly divided regions were required for quantitative analysis using ImageJ software. All immunofluorescence assays were repeated at least three times.

\section{Kartogenin injection in mice}

We followed the methods of Lijuan Zhou et al. $2018[23,24]$ to induce anagen phase in female C57BL/ 6 mice. The mice were then randomized into vehicle (DMSO)- and kartogenin-treated groups ( $\mathrm{n}=6$ each). To examine the anagen-to-catagen transition, mice were treated with $100 \mu \mathrm{L}$ kartogenin $(10 \mu \mathrm{M})$ dissolved in DMSO or vehicle (DMSO) once daily from p.d. 12-17 (total $600 \mu \mathrm{L}$ ) and sacrificed at p.d. 18 and p.d.21. Skin samples were collected for analysis and regrown hairs were plucked from depilated dorsal region at p.d.18 and p.d.21. We measured the average hair length from 30 hairs per mouse.

\section{Quantitative histomorphometry}

Dorsal skin samples were fixed with formaldehyde solution and sectioned into 5-7 $\mu \mathrm{m}$ sections, which were stained with hematoxylin and eosin (HE), followed by analysis of hair length, hair bulb diameter, hair cycling score (HCS), and skin thickness using a light microscope. Based on previous studies [25], we assigned scores of 100, 200, and 300 for anagen VI, catagen II-III, and catagen IV-V phases HFs during anagen-to-catagen transition, respectively [26]. At least $50 \mathrm{HFs}$ per sample were analyzed.

\section{Statistical analysis}

All experiments were repeated at least three times. Statistical analysis was performed using SPSS software (ver. 17.0; SPSS Inc., Chicago, IL, USA). Results were compared using Student's $t$-tests and one-way ANOVA. Results are presented as mean \pm standard deviation (SD).

\section{Results}

\section{Kartogenin stimulated proliferation and increased S, G2, and S/G1 phases in ORSCs in vitro}

Kartogenin (Fig. 1a) (0.5, and $1 \mu \mathrm{M})$ promoted ORSC proliferation in a concentration-dependent manner compared with untreated control cells (Fig. 1b) $(\mathrm{P}<0.01)$. We then analyzed the effect of $1 \mu \mathrm{M}$ kartogenin on the cell cycle status in ORSCs by propidium iodide-RNase staining and flow cytometry. The results showed that $41.88 \%$ of ORSCs entered the $S$ and $G 2$ phases following treatment with $1 \mu \mathrm{M}$ kartogenin, compared with only $33.64 \%$ of vehicle-treated cells $(\mathrm{P}<0.01)$ (Fig. 1c, d). Treatment with kartogenin $1 \mu \mathrm{M}$ resulted in 1.32 -fold $(\mathrm{P}<0.01)$ increases in the S-phase fraction and 1.50-fold $(\mathrm{P}<0.01)$ increases in the S/G1 fraction, respectively (Fig. 1e). These results demonstrated that kartogenin stimulated ORSC proliferation. 

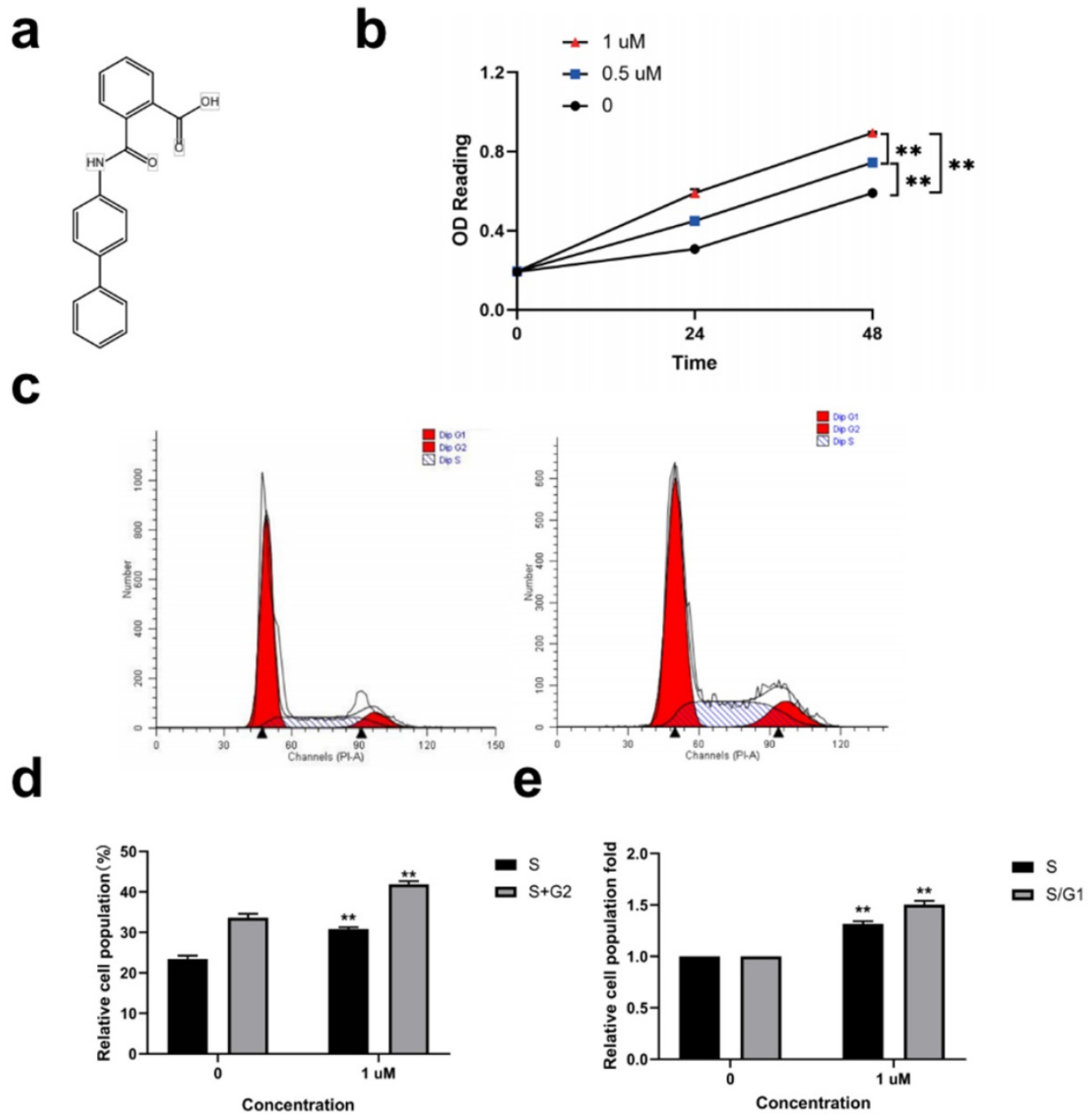

Figure 1. Kartogenin treatment stimulated human ORSC proliferation and increased cells in S and S/GI phases in vitro. a. Structure of kartogenin. b. Kartogenin increased proliferation of ORSCs in a dosedependent manner, as measured by MTS assay. c, d. Distributions of ORSCs in G1, S, and G2 phases in the presence or absence of kartogenin, detected by flow cytometry. e. Fold changes in the fractions of cells in S and S/G1 phases with or without kartogenin treatment. Bars represent mean \pm SD. $* * \mathrm{P}<0.01$

\section{Kartogenin stimulated ORSC migration function in vitro}

Kartogenin has been shown to coordinate cellular migration and proliferation during cartilage regeneration [12,15]. We therefore determined if kartogenin stimulation increased ORSC migration and wound closure using in vitro Transwell ${ }^{\circledR}$ and scratch assays, respectively. Kartogenin $1 \mu \mathrm{M}$ promoted ORSC migration relative to untreated control cells $(\mathrm{P}<0.01)$, as demonstrated by wound healing assays (Fig. 2a-c) and Transwell ${ }^{\circledR}$ (Fig. 2d-e).

\section{Kartogenin downregulated expression of TGF- $\beta 2 / S m a d$ signaling molecules in ORSCs in vitro}

P-Smad2/p-Smad3 form a heteromeric complex with Smad4, which then translocate to the nucleus and regulates the expression of several genes [7]. We examined the mRNA and protein levels of TGF- $\beta 2 /$ Smad signaling molecules in ORSCs treated with kartogenin for $24 \mathrm{~h}$, using RT-PCR and western blotting, respectively. Kartogenin treatment decreased TGF- $\beta 2$, p-Smad2, and p-Smad3 protein expression levels in dose-dependent manners (Fig. 3d, Sup Fig. 1). At a concentration of $1 \mathrm{uM}$, the protein level of TGF- $\beta 2$, p-Smad2, and p-Smad3 were decreased $0.66-, 0.49-, 0.67$-fold $(\mathrm{P}<0.01)$, respectively, compared with control cells (Fig. 3e-g). Similar trends were observed in mRNA levels of TGF- $\beta 2$, Smad2, and Smad3, which were decreased 0.75-fold $(\mathrm{P}<0.05)$, 0.76 -fold $(\mathrm{P}<0.01)$, and 0.68 -fold $(\mathrm{P}<0.01)$, respectively (Fig. 3a-c). The localization of TGF- $\beta 2$ in ORSCs after treatment with 0 or $1 \mu \mathrm{M}$ kartogenin for $24 \mathrm{~h}$ was determined by cellular fluorescence. TGF- $\beta 2$ was expressed in both the nuclei and cytoplasm of 
untreated control ORSCs, most notably in the cytoplasm (Fig. 3h), while p-Smad2 and p-Samd 3 also showed strong nuclei signals (Fig. 3i-j). In contrast, TGF- $\beta 2$, p-Smad2, and p-Smad3 expression levels were all reduced after treatment with kartogenin (Fig. $3 e-g)$. The results of quantitative analyses of TGF- $\beta 2$, p-Smad2, and p-Smad3 were consistent with the above results. a

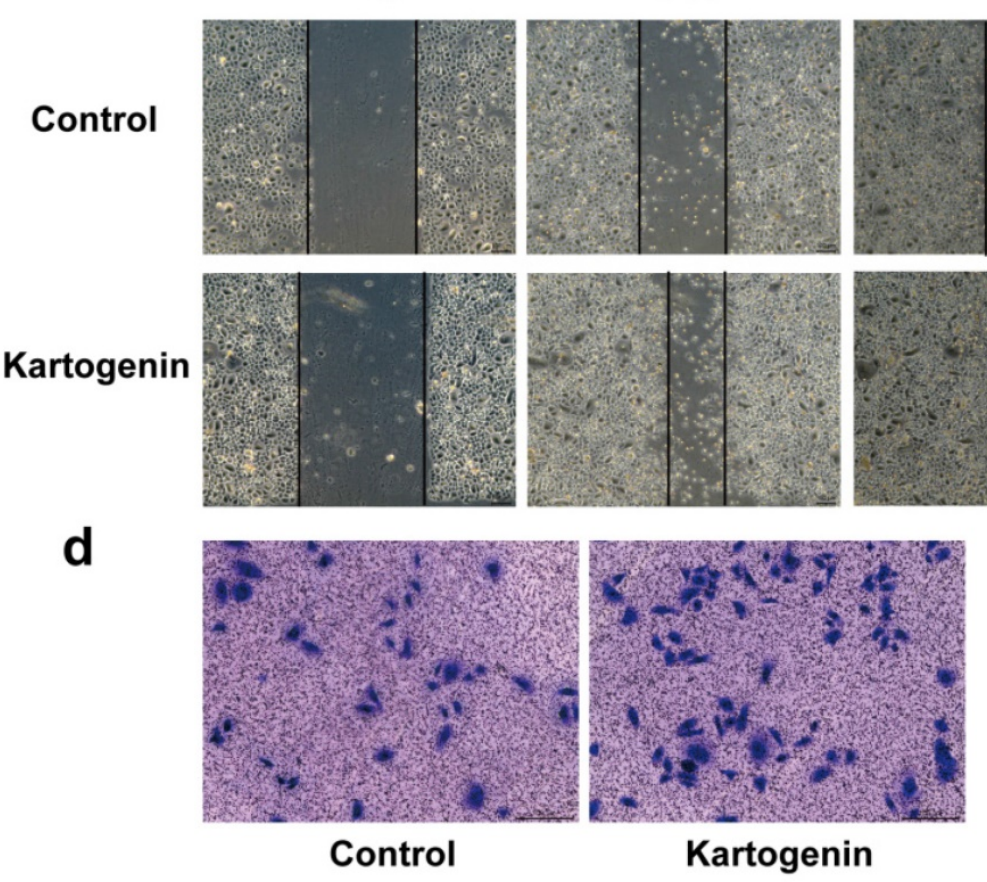

$24 \mathrm{~h}$

b

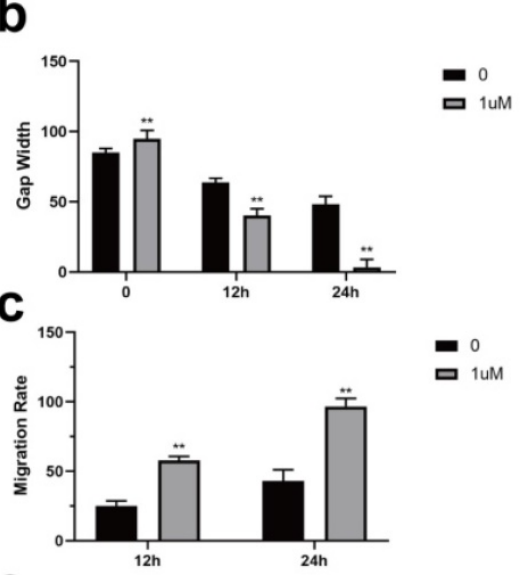

e

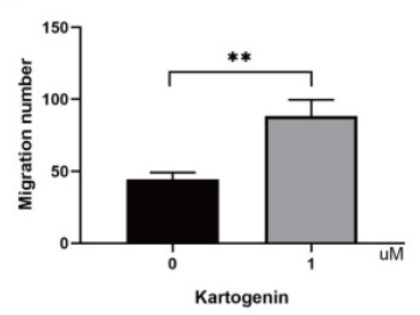

Figure 2. Kartogenin increased human ORSC migration function. a-c. Wound scratch assays of ORSCs cultured with or without kartogenin treatment. d-e. Transwell assays of ORSCs with or without kartogenin treatment. Data represent mean \pm SD. $* * P<0.01$.

a

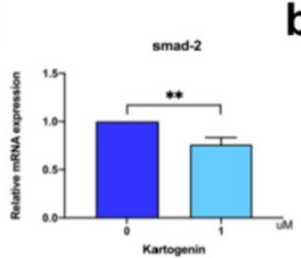

b

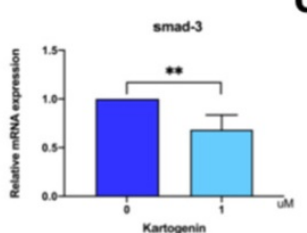

C

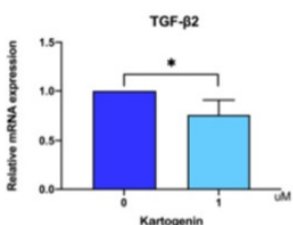

d

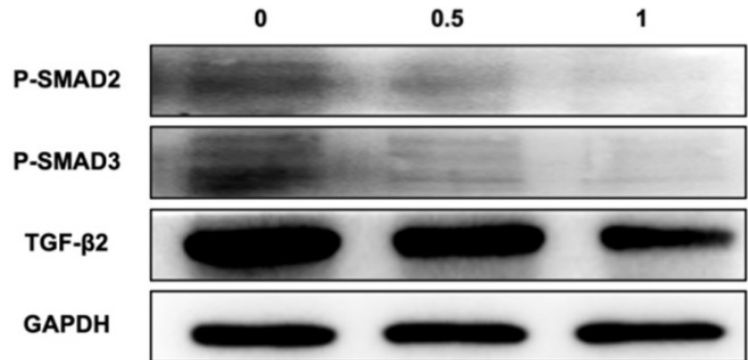

e

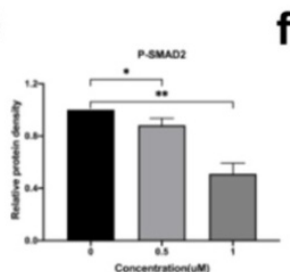

f

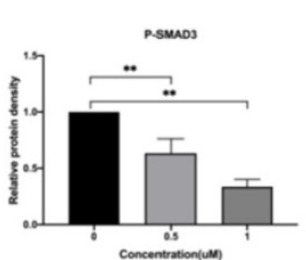

g

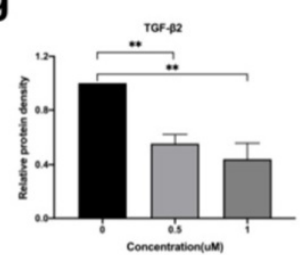

h

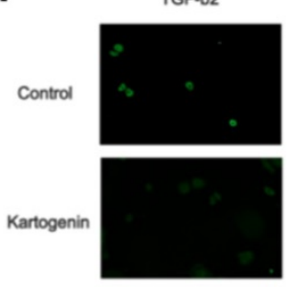

i

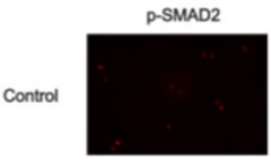

50KD

37KD
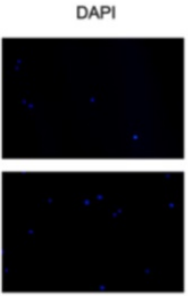

DAPI
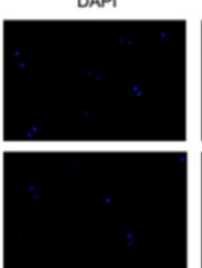

DAPI

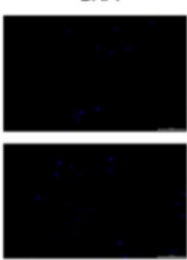

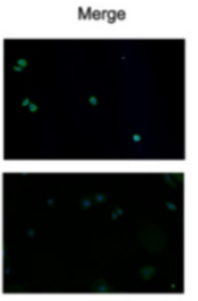

Merge
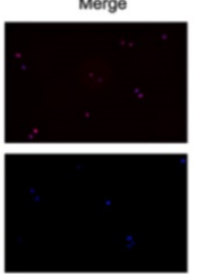

j

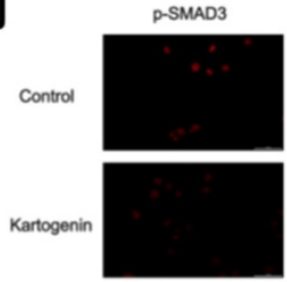

Merge

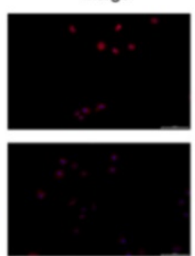

Figure 3. Kartogenin reduced the mRNA and protein levels of TGF- $\beta 2 / S m a d$ signaling molecules. a-c. Relative mRNA expression levels of TGF- $\beta 2$, Smad2, and Smad3 in human ORSCs, determined by RT-PCR. d. TGF- $\beta 2$, p-Smad, 2 and p-Smad3 protein expression levels in human ORSCs, detected by western blotting. The grouping of blots was cropped from different gels. e-g. Quantitative analysis of TGF- 32 , p-Smad2, and p-Smad3 protein levels. h-j TGF- $\beta 2$ shown in green, p-Smad2, and p-Smad3 shown in red and nuclei counterstained with DAPI (blue). Merged images indicate the expression and location of TGF- $\beta 2, p-S m a d 2$, and $p-S m a d 3$.*P $<0.05$, **P $<0.01$. 


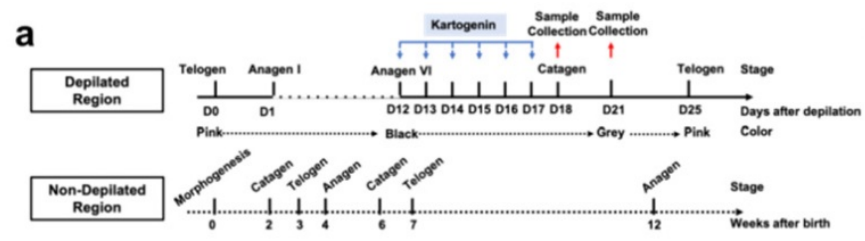

\section{d}

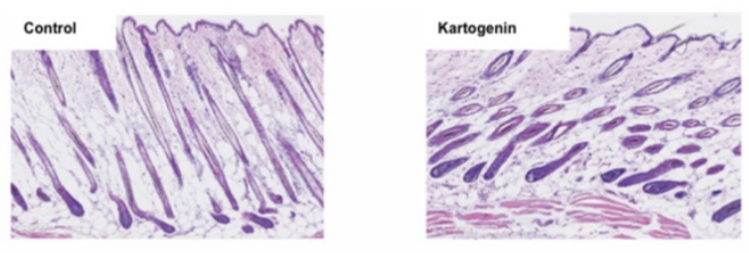

b
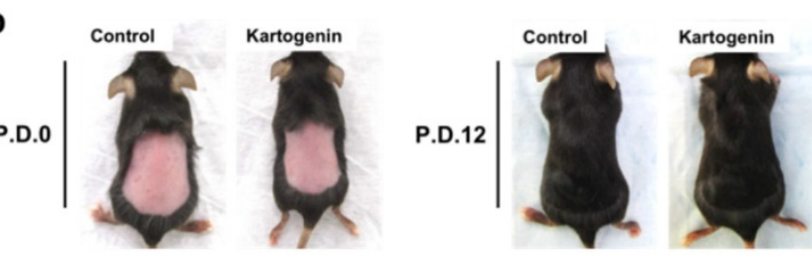

C
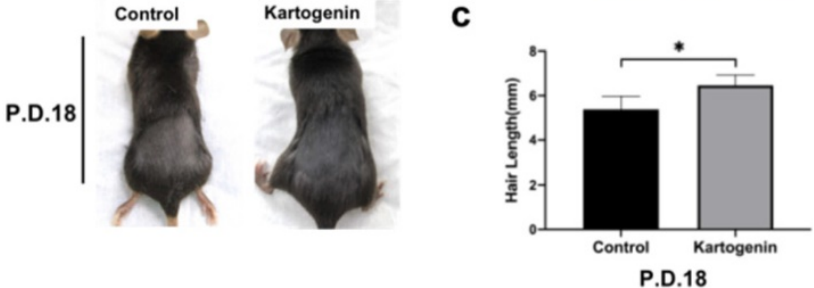

e

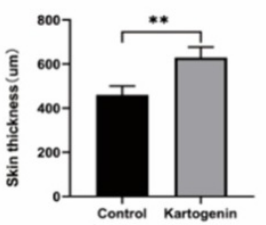

g

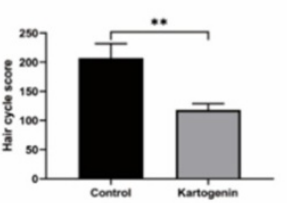

f

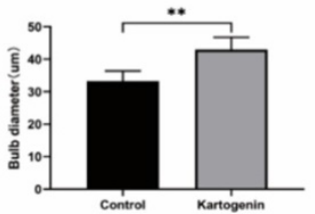

h

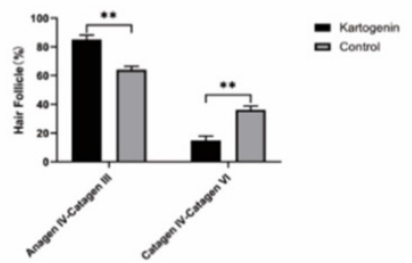

Figure 4. Injection of kartogenin delayed transition of mouse HFs from anagen phase to catagen phase. Kartogenin $(0,1 \mu \mathrm{M}$ in $100 \mu \mathrm{L})$ was injected subcutaneously into mouse dorsal skin at p.d. $12(100 \mu \mathrm{L}$ over 6 days, total $600 \mu \mathrm{L})$. Results represent mean \pm SD ( $=6$ per group). $* \mathrm{P}<0.05$, $* * \mathrm{P}<0.01$. a. Experimental time course. b. Macrophotograph of control group and kartogenin group at p.d.0, p.d.12 and p.d.18. c. Hair length in dorsal skin. d. H\&E staining images of control group and kartogenin group. e. Skin thickness. f. Bulb diameter. g. HCS. h. HFs (\%). Scale bar: $100 \mu \mathrm{m}$.

Taken together, these results thus demonstrated that kartogenin downregulated the TGF- $\beta 2 / \mathrm{Smad}$ signaling pathway.

\section{Local injection of kartogenin into HFs in anagen phase delayed entry into catagen phase in vivo}

We investigated the effect of kartogenin on the anagen-to-catagen transition in mice HFs in vivo. Mice that underwent depilation to induce anagen phase, were treated with kartogenin $(10 \mu \mathrm{M})$ or vehicle by subcutaneously injection from p.d.12 to p.d.17, and then their HF morphology at p.d.18 and p.d.21 were examined (Fig. 4a). The changes of mice dorsal skin in each group were analyzed at p.d.0, p.d.12, p.d.18. Our results indicated that the administration of kartogenin signifitantly prolonged the anagen phase compared with control group (Fig. 4b). Further we measured the length of refreshed hair from each group at p.d.18, and the kartogenin group showed longer hair than control mice at p.d.18 $(\mathrm{p}<0.05)$ (Fig 4c). Histomorphometric analysis of H\&E -stained tissue sections revealed that kartogenin-treated HFs (Fig. 4d) were in anagen phase VI, with significantly larger bulb diameter and thicker skin compared with the control group $(\mathrm{P}<0.01)$ (Fig. $4 \mathrm{~d}-\mathrm{f})$. We also calculated HCS and HF (\%) for a more accurate determination of the hair growth cycle stage. The total HCS was lower in the kartogenin compared with the control group $(\mathrm{P}<0.01)$ (Fig. 4g), with $14.86 \%$ of HFs in the catagen IV-VI stage in the treated vs. approximately $36.13 \%$ in the control group (Fig. 4h).

To better know the effects of kartogenin on hair growth, we further collected the dorsal skin sample at p.d.21 (Fig. 5), we found that at p.d.21, HFs of the control group were mainly in late catagen phase while HFs of kartogenin group just started to enter into early catagen phase (Fig. 5a, 5c-g). Moreover, we measured the length of new hairs of the depilated region at p.d.18 (Fig. 4c) and p.d.21 (Fig. 5c), we found out that injection of kartogenin resulted in longer hair shaft both at p.d.18 and p.d.21 by 1.20 -fold and 1.32 -fold higher respectively $(p<0.05)$. These results indicated that kartogenin could delay anagen-tocatagen transition and promote hair regeneration.

\section{Discussion}

The results of this study demonstrated that kartogenin regulated HF cell growth and the hair growth cycle transition. Kartogenin stimulated the proliferation and migration of cultured human ORSCs in vitro, which are necessary steps in HF growth and development. Furthermore, direct injection of kartogenin into murine HFs in anagen phase delayed catagen phase progression, accompanied by longer HF shafts, larger bulbs, and thicker skin. These effects were accompanied by downregulation of TGF- $\beta 2$ / Smad signaling pathway molecules.

To evaluate the efficacy of kartogenin in vivo, anagen phase was induced in C57BL/ 6 mice by depilation, according to a widely used model [29], and kartogenin was then injected subcutaneously at 
a

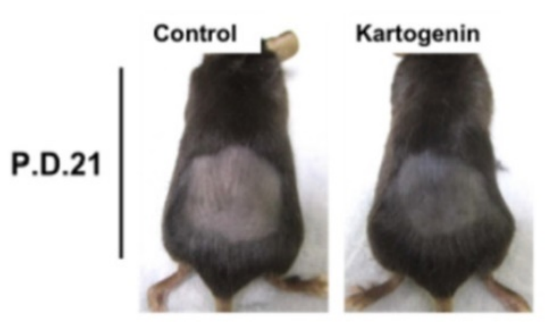

b

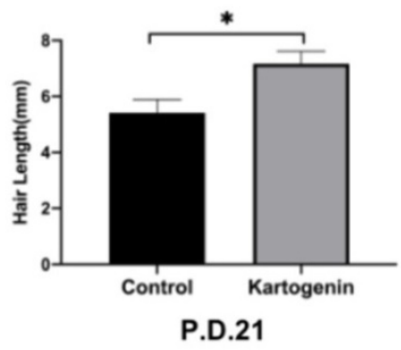

C

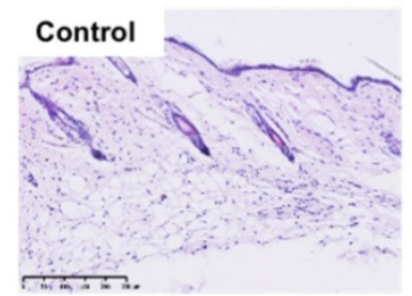

d

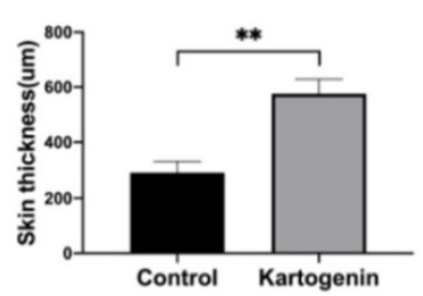

f

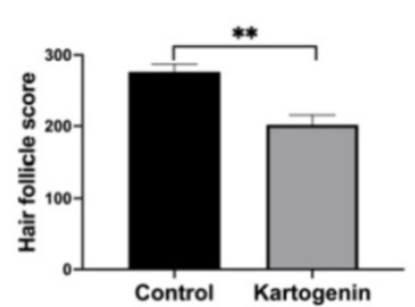

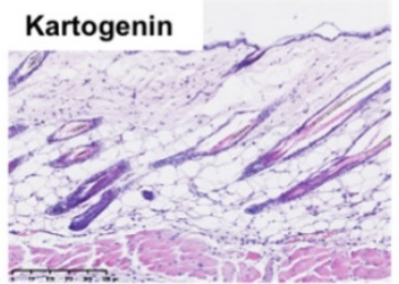

e

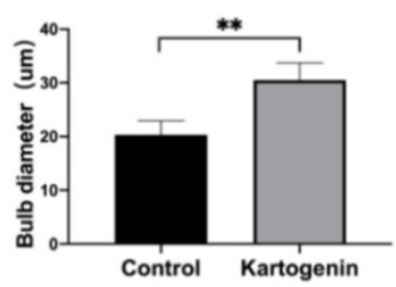

g

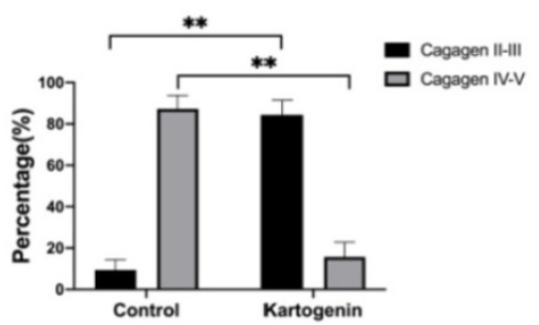

Figure 5. Injection of kartogenin prolonged anagen phase and increased hair length. a. Macrophotograph of control group and kartogenin group at p.d.21. b. Hair length. c. H\&E staining images of control group and kartogenin group skin sample at p.d.21. d. Skin thickness. e. Bulb diameter. f. HCS. g. HFs (\%). Scale bar: 100 mm. Results represent mean $\pm S D(n=6$ per group). $* P<0.05$, **P $<0.01$.

depilation-induced anagen phase time point (p.d. 12). Histological analysis showed that kartogenin delayed the anagen-to-catagen transition, thus prolonging the anagen phase and enlonged the length of hair shafts. Both men and women would experience hair loss $[30,31]$. Burg et al. demonstrated a reduction in the proportion of HFs in anagen phase in patients with AGA and female pattern hair loss (FPHL) [30]. Our current in vitro findings demonstrated that kartogenin promoted hair cell growth and migration in a dose-dependent manner. The behaviors of ORSCs are mainly influenced by rapid remodeling of epithelial keratinocytes [30] and are critical characteristics of the hair anagen-to-catagen transition, showing high proliferation and migration capacities during anagen phase and apoptotic features in catagen phase [32]. These features suggested that kartogenin may be effective in hair loss by prolonging the anagen phase.

Injection of mice with kartogenin in the anagen phase delayed entry into catagen phase, similar to the effect on HFs in TGF- $\beta$-null mice, which increased hair cell proliferation and morphological suppression of catagen phase with longer anagen phase [33]. Based on the effects of kartogenin on human ORSCs and the prolongation of anagen phase in mice, we hypothesized that TGF- $\beta 2$ might be involved in kartogenin-induced anagen phase maintenance and hair growth cycle transitions [27].

To the best of our knowledge, the effects of kartogenin on TGF- $\beta 2$ signaling have not been investigated previously. TGF- $\beta 2$ signaling is a key signaling pathway in HF growth and development, through suppressing epithelial cell growth $[19,21,34]$ and regulating apoptosis via $\mathrm{p}-\mathrm{Smad} 2$ and $\mathrm{p}-\mathrm{Smad} 3$ [20]. Furthermore, as a catagen phase-inducer, TGF- $\beta 2$ inhibits hair growth and development by reducing anagen phase and accelerating the transition from anagen phase into catagen phase [19, 27, 34]. Suppression of TGF- $\beta 2$ and $p-S m a d 2 / 3$ activities thus prolongs the anagen phase and increases hair cell proliferation [34, 35]. Inhibition of TGF- $32 / \mathrm{Smad}$ signaling is therefore a well-accepted method of treating most hair loss types, including alopecia induced by drugs, androgens, and psychological factors. The results of the present study revealed that kartogenin downregulated TGF- $\beta 2$ mRNA and protein expression levels in cultured human ORSCs, and also downregulated other TGF- $\beta 2 / S m a d$ signaling pathway molecules in a dose-dependent manner.

Kartogenin possesses several advantages as a potential therapy for alopecia. It has shown excellent 
biocompatibility [12] and has demonstrated no toxicity in various cell types and no obvious adverse effects in animals [11, 12, 36]. Furthermore, kartogenin is a highly stable, non-protein small molecule that can be cheaply and easily synthesized [36], and which can be easily stored and transported at room temperature $[6,12]$. In addition, similar to finasteride, which is the most widely used oral drug for androgenic alopecia, kartogenin could also suppress the anagen-to-catagen phase transition in HFs to prolong anagen phase [37], so a possible additive or synergy effect may exist between finasteride and kartogenin. Kartogenin is thus a promising new drug for the treatment of anagen-reduced hair loss. The results of this study provided novel evidence for the function of kartogenin in regulating HF growth and hair growth cycle transition.

\section{Supplementary Material}

Supplementary figure.

https://www.medsci.org/v19p0537s1.pdf

\section{Acknowledgements}

\section{Funding}

This work was supported by the National Natural Science Foundation of China under Grant No. 81972959.

\section{Data Sharing Statement}

The data used to support the findings of this study are available from the corresponding author upon request.

\section{Competing Interests}

The authors have declared that no competing interest exists.

\section{References}

1. Stenn K, Paus R. Controls of hair follicle cycling Physiol. Rev. 2001;81(1): 449-494.

2. Al-Nuaimi $Y$, Hardman JA, Biro $T$, et al. A meeting of two chronobiological systems: circadian proteins Period1 and BMAL1 modulate the human hair cycle clock J. Invest. Dermatol. Mar 2014;134(3):610-619.

3. Hawkshaw NJ, Hardman JA, Haslam IS, et al. Identifying novel strategies for treating human hair loss disorders: Cyclosporine A suppresses the Wnt inhibitor, SFRP1, in the dermal papilla of human scalp hair follicles PLoS Biol. May 2018;16(5):e2003705.

4. Naruse T, Aoki M, Fujimoto N, et al. Novel ALK5 inhibitor TP0427736 reduces TGF-betainduced growth inhibition in human outer root sheath cells and elongates anagen phase in mouse hair follicles Pharmacol. Rep. Jun 2017;69(3):485-491.

5. Midorikawa T, Chikazawa T, Yoshino T, Takada K, Arase S. Different gene expression profile observed in dermal papilla cells related to androgenic alopecia by DNA macroarray analysis J. Dermatol. Sci. Oct 2004;36(1):25-32.

6. Strazzulla LC, Wang EHC, Avila L, et al. Alopecia areata: An appraisal of new treatment approaches and overview of current therapies J. Am. Acad. Dermatol. Jan 2018;78(1):15-24.

7. Phillips TG, Slomiany WP, Allison R. Hair Loss: Common Causes and Treatment Am. Fam. Physician. Sep 15 2017;96(6):371-378.
8. Adil A, Godwin M. The effectiveness of treatments for androgenetic alopecia: A systematic review and meta-analysis J. Am. Acad. Dermatol. Jul 2017;77(1):136-141.e135.

9. Mori $\mathrm{O}$, Uno $\mathrm{H}$. The effect of topical minoxidil on hair follicular cycles of rats J. Dermatol. May 1990;17(5):276-281.

10. Wang J, Zhou J, Zhang N, Zhang X, Li Q. A heterocyclic molecule kartogenin induces collagen synthesis of human dermal fibroblasts by activating the smad4/smad5 pathway Biochem. Biophys. Res. Commun. Jul 18 2014;450(1):568-574.

11. Johnson K, Zhu S, Tremblay MS, et al. A stem cell-based approach to cartilage repair Science. May 11 2012;336(6082):717-721.

12. Li X, Ding J, Zhang Z, et al. Kartogenin-Incorporated Thermogel Supports Stem Cells for Significant Cartilage Regeneration ACS applied materials \& interfaces. Mar 2 2016;8(8):5148-5159.

13. Zhang J, Yuan T, Zheng N, Zhou Y, Hogan MV, Wang JH. The combined use of kartogenin and platelet-rich plasma promotes fibrocartilage formation in the wounded rat Achilles tendon entheses Bone \& joint research. Apr 2017;6(4):231-244.

14. Zhang J, Wang JH. Kartogenin induces cartilage-like tissue formation in tendon-bone junction Bone research. 2014;2.

15. Decker RS, Koyama E, Enomoto-Iwamoto M, et al. Mouse limb skeletal growth and synovial joint development are coordinately enhanced by Kartogenin Dev. Biol. Nov 15 2014;395(2):255-267.

16. Limat A, Hunziker T. Use of epidermal equivalents generated from follicular outer root sheath cells in vitro and for autologous grafting of chronic wounds Cells Tissues Organs. 2002;172(2):79-85.

17. Jahoda C, Reynolds A. Skin stem cells - a hairy issue Nat. Med. Oct 2000;6(10):1095-1097.

18. Kim CD, Choe Y, Shim C, Kim K. Interferon Beta secreted from human hair dermal papilla cells inhibits the growth of outer root sheath cells cultured in vitro Biochem. Biophys. Res. Commun. Jan 25 2002;290(3):1133-1138.

19. Sasajima M, Moriwaki S, Hotta M, Kitahara T, Takema Y. trans-3,4'-Dimethyl-3-hydroxyflavanone, a hair growth enhancing active component, decreases active transforming growth factor beta2 (TGF-beta2) through control of urokinase-type plasminogen activator (uPA) on the surface of keratinocytes Biol. Pharm. Bull. Mar 2008;31(3):449-453.

20. Paus R, Foitzik K, Welker P, Bulfone-Paus S, Eichmuller S. Transforming growth factor-beta receptor type I and type II expression during murine hair follicle development and cycling J. Invest. Dermatol. Oct 1997;109(4):518-526.

21. Tsuji Y, Denda S, Soma T, Raftery L, Momoi T, Hibino T. A potential suppressor of TGF-beta delays catagen progression in hair follicles J. Investig. Dermatol. Symp. Proc. Jun 2003;8(1):65-68.

22. Song K, Wang H, Krebs TL, Kim SJ, Danielpour D. Androgenic control of transforming growth factor-beta signaling in prostate epithelial cells through transcriptional suppression of transforming growth factor-beta receptor II Cancer Res. Oct 1 2008;68(19):8173-8182.

23. Zhou L, Jing J, Wang H, Wu X, Lu Z. Decorin promotes proliferation and migration of ORS keratinocytes and maintains hair anagen in mice. Experimental dermatology 2018;27:1237-1244.

24. Zhou L, Wang H, Jing J, Yu L, Wu X, Lu Z. Morroniside regulates hair growth and cycle transition via activation of the Wnt/ $\beta$-catenin signaling pathway. Scientific reports 2018;8:13785.

25. Jing J, Wu XJ, Li YL, Cai SQ, Zheng M, Lu ZF. Expression of decorin throughout the murine hair follicle cycle: hair cycle dependence and anagen phase prolongation Exp. Dermatol. Jul 2014;23(7):486-491.

26. Kwack MH, Kim MK, Kim JC, Sung YK. Dickkopf 1 promotes regression of hair follicles J. Invest. Dermatol. Jun 2012;132(6):1554-1560.

27. Muller-Rover S, Handjiski B, van der Veen C, et al. A comprehensive guide for the accurate classification of murine hair follicles in distinct hair cycle stages J. Invest. Dermatol. Jul 2001;117(1):3-15.

28. AJ K, M T. Mammalian target of rapamycin complex 1 (mTORC1) may modulate the timing of anagen entry in mouse hair follicles Exp. Dermatol. 2013;22(1):77-80.

29. Amberg N, Holcmann M, Stulnig G, Sibilia M. Effects of Imiquimod on Hair Follicle Stem Cells and Hair Cycle Progression J. Invest. Dermatol. Nov 2016;136(11):2140-2149.

30. Burg D, Yamamoto M, Namekata M, Haklani J, Koike K, Halasz M. Promotion of anagen, increased hair density and reduction of hair fall in a clinical setting following identification of FGF5-inhibiting compounds via a novel 2-stage process Clin. Cosmet. Investig. Dermatol. 2017;10:71-85.

31. Park S, Erdogan S, Hwang D, Hwang S, Han EH, Lim YH. Bee Venom Promotes Hair Growth in Association with Inhibiting 5alpha-Reductase Expression Biol. Pharm. Bull. Jun 1 2016;39(6):1060-1068. 
32. Huang W, Lai S, Chiu H, et al. Mobilizing Transit-Amplifying Cell-Derived Ectopic Progenitors Prevents Hair Loss from Chemotherapy or Radiation Therapy Cancer Res. 2017;77(22):6083-6096.

33. Soma T, Dohrmann CE, Hibino T, Raftery LA. Profile of transforming growth factor-beta responses during the murine hair cycle J. Invest. Dermatol. Nov 2003;121(5):969-975.

34. Soma $\mathrm{T}$, Tsuji $\mathrm{Y}$, Hibino $\mathrm{T}$. Involvement of transforming growth factor-beta2 in catagen induction during the human hair cycle J. Invest. Dermatol. Jun 2002;118(6):993-997.

35. Lefever T, Pedersen E, Basse A, et al. N-WASP is a novel regulator of hair-follicle cycling that controls antiproliferative TGF\{beta\} pathways J. Cell Sci. Jan 1 2010;123(Pt 1):128-140.

36. Im GI. Application of kartogenin for musculoskeletal regeneration Journal of biomedical materials research. Part A. Apr 2018;106(4):1141-1148.

37. Danesh MJ, Murase JE. Further research needed if finasteride is to become standard of care for frontal fibrosing alopecia (FFA) J. Am. Acad. Dermatol. Apr 2016;74(4):e75 